\title{
A Comprehensive Analysis on Interference Avoidance for Dynamic Spectrum Sharing between FDD LTE and NR
}

\author{
Jun Wang $\mathbb{D}^{1}$ and Dejun $M u \mathbb{D}^{1,2}$ \\ ${ }^{1}$ Northwestern Polytech University, Xi'an, 710000, China \\ ${ }^{2}$ Research \& Development Institute of Northwestern Polytechnical University, Shenzhen 518057, China \\ Correspondence should be addressed to Jun Wang; 648051693@qq.com
}

Received 27 October 2021; Revised 18 November 2021; Accepted 20 December 2021; Published 18 January 2022

Academic Editor: Narasimhan Venkateswaran

\begin{abstract}
Copyright ( 92022 Jun Wang and Dejun Mu. This is an open access article distributed under the Creative Commons Attribution License, which permits unrestricted use, distribution, and reproduction in any medium, provided the original work is properly cited.
\end{abstract}

\begin{abstract}
This paper presents a comprehensive analysis on the interference avoidance for dynamic spectrum sharing (DSS) between FDD LTE and NR to solve the coverage layer for NR standalone (SA) network with low FDD LTE bands. In the first place, detailed collisions between FDD LTE and NR are presented and involved with physical control channel, physical data channel, and physical signal of both downlink and uplink. The corresponding principle of collision avoidance is also proposed based on the flexible configuration of NR physical layer in both the time domain and the frequency domain. Due to LTE CRS across the whole bandwidth, the collide with LTE CRS cannot be fully avoided by flexible configuration and need LTE CRS puncturing or NR puncturing on these collided LTE CRS with DSS activation. However, both LTE CRS puncturing and NR puncturing more or less cause the impacts on the performance. As with NR puncturing for LTE CRS, the simulation analysis on the impacts of NR SSB and RMSI is presented. On the other hand, LTE CRS puncturing influences the reception performance of LTE UE, and we analyze the impact of RSRP measurement, demodulation, and frequency/time offset measurement by simulation. Finally, capacity loss also is explained based on essential overhead.
\end{abstract}

\section{Introduction}

Both capacity and coverage are essential for each generation mobile network, especially in the initial deployment [1]. Undoubtedly, 5G coverage layer for outside cities will be a challenge for operators. Low bands are the best choices for $5 \mathrm{G}$ coverage layer, but most accessible low bands are occupied by existing technology [2]. Few options are left for operators. $600 \mathrm{MHz}$ or $700 \mathrm{MHz}$ is the most possible spectrum for $5 \mathrm{G}$ coverage layer, but nationwide deployment will be tremendous investment for operators. On the other hand, refarming existing spectrum to $5 \mathrm{G}$ is a helpless choice for operators where these low bands are still occupied. Undoubtedly, the refarming solution will decrease the capacity of existing network while LTE traffic domains continue to grow in the next these years till the penetration of 5G User Equipment (UE) exceeds LTE UE. Therefore, a coefficiency spectrum refarming solution is urgent for operators to solve both $5 \mathrm{G}$ coverage lay and LTE capacity.
Dynamic spectrum sharing (DSS) between LTE and NR is proposed to satisfy operators' requirements, and it allows that one dedicated carrier can support LTE and NR simultaneously by partially or fully overlapped carriers [3-6]. The foundation of DSS is the flexible configuration of $5 \mathrm{G}$ physical layer, and NR signals will be assigned to unused LTE resources as far as possible. Undoubtedly, interference avoidance will be the first issue to focus.

In this paper, we aim at a comprehensive analysis on interference avoidance for DSS between FDD LTE and NR based on model simulation. In the paper [7], it gives the general guideline for DSS and requires the implementation of DSS for standalone (SA) and nonstandalone (NSA) following the same principles. Because NSA is temporary, DSS on SA will be our focus. In doing the analysis, the interference of downlink physical channels and uplink physical channels between FDD LTE and NR is distinguished, and the principle of interference avoidance is subsequently presented in details. Based on the principle, puncturing on 
TABLe 1: Downlink interference conflict between FDD LTE and NR.

\begin{tabular}{lccccccc}
\hline NR/LTE & PDSCH & $\begin{array}{c}\text { PDCCH } \\
\text { PCFICH } \\
\text { PHICH }\end{array}$ & CRS & DMRS & $\begin{array}{c}\text { CSI- } \\
\text { RS }\end{array}$ & PBCH & $\begin{array}{c}\text { PSS } \\
\text { SSS }\end{array}$ \\
\hline PDSCH & $\sqrt{ }$ & $\sqrt{ }$ & $\sqrt{ }$ & $\sqrt{ }$ & $\sqrt{ }$ & $\sqrt{ }$ \\
CORESET & $\sqrt{ }$ & $\sqrt{ }$ & & & & & \\
DMRS & & & $\sqrt{ }$ & $\sqrt{ }$ & & \\
CSI-RS & $\sqrt{ }$ & & & & $\sqrt{ }$ & \\
SSB & $\sqrt{ }$ & & $\sqrt{ }$ & $\sqrt{ }$ & $\sqrt{ }$ & $\sqrt{ }$ \\
RMSI & $\sqrt{ }$ & & $\sqrt{ }$ & $\sqrt{ }$ & $\sqrt{ }$ & $\sqrt{ }$ \\
TRS & $\sqrt{ }$ & & $\sqrt{ }$ & $\sqrt{ }$ & $\sqrt{ }$ & $\sqrt{ }$ \\
\hline
\end{tabular}

symbols is the best choice for DSS and LTE CRS (Cell Reference Signal) puncturing and NR puncturing for LTE CRS presented for DSS. As with LTE CRS puncturing, the simulation is focused on the impact on RSRP (Reference Signal Receiving Power), demodulation, time-domain, and frequency-domain measurement. For NR puncturing, SNR (Signal Noise Ration) is our focus.

We present the paper in seven sections. In Sections 2 and 3 , downlink and uplink interference conflict between FDD LTE and NR is presented for both control channels and data channels and detailed avoidance principle and mechanism are also introduced for DSS. Section 4 covers analysis on the impact on NR SSB and RMSI with NR puncturing in DSS based on AGWN and TDL-A. Subsequently, in Section 5, the performance on LTE CRS puncturing for NR is evaluated based on RSRP measurement, demodulation, and frequency/ time offset measurement. As with capacity loss, it is mentioned based on systematic overhead in Section 6.

\section{Downlink Interference Avoidance Principle}

The subcarrier spacing (SCS) used for Synchronization Signal and $\mathrm{PBCH}$ Block (SSB) is dependent on frequency band; it can be either $15 \mathrm{kHz}$ or $30 \mathrm{kHz}$ for FR1 [8]. $15 \mathrm{kHz}$ is the best choice for DSS between FDD LTE and NR because the energy of $30 \mathrm{kHz}$ subcarriers leaks to $15 \mathrm{kHz}$ subcarriers and causes interference if $30 \mathrm{kHz}$ subcarriers are adopted for NR.

Downlink interference avoidance needs consider interference on physical channels and signals. As shown in Table 1, these interferences caused by these channels and signals need to be avoided when DSS are activated. From the LTE perspective, the downlink physical channel and signals of LTE for interference avoidances include PDSCH, PDCCH, PCFICH, PHICH, CRS, DM-RS, CSI-RS, PBCH, SSS, and PSS [9]. Similarly, PDSCH, PDCCH, CRS, CSIRS, $\mathrm{PBCH}$, SSS, and PSS are also defined for NR physical channels and signals and need to be considered for interference avoidance, but there are some differences between LTE and NR on the definitions of physical channels and signals [10]. divided into control region and data region, as shown in Figure 1. Control region occupies the first 4 symbols of each subframe; in other words, the control region is across the whole bandwidth in the frequency domain. Generally, the control region occupies the first 3 symbols except $1.4 \mathrm{MHz}$ LTE carrier.

LTE control region consists of PCFICH, PDCCH, and PHICH. PCFICH stands for Physical Control Format Indicator Channel. It carries CFI (Control Format Indicator), which indicates the number symbols that can be used for $\mathrm{PDCCH}$ and PHICH and occupies the first symbol in the control region. Based on the value of CFI, PDCCH occupies the corresponding symbols in control regions. As with $\mathrm{PHICH}$, it stands for Physical channel HybridARQ Indicator Channel and occupies the first symbol in control region.

For NR control signaling, CORESET (Control Resource Set) is defined to carry PDCCH/DCI. In other words, it is equivalent to the LTE control region. But it is still different from the LTE control region in configurations and parameters.

Although NR UE obtains SSB information, the information carried by MIB is not enough to keep UE camp cell and initiate further access and UE still needs certain essential information [11]. Therefore, the essential information is divided into two parts: one part is carried by MIB, and the other is carried by RMSI (Remaining Minimum System Information). CORESET 0 is defined to indicate RMSI position and is configured by MIB. Meanwhile, paging, OSI, MSG2/MSG3, and retransmission/MSG4 are also indicated by CORESET 0 .

The position of CORESET is very complicated. Following 3GPP's rules of CORESET 0 for FR1, the multiplexing pattern of SSB and CORESET adopts Pattern 1, which belongs to TDM. CORESET 0 occupies 3 symbols maximally in the time domain and $48 \mathrm{RBs}$ at least if CORESET is transmitted on 1 symbol.

Based on the above definitions of LTE control region and NR CORESET 0, the interference between LTE PDCCH/ $\mathrm{PCFICH} / \mathrm{PHICH}$ and NR CORESET 0 can be avoided in the frequency domain. NR CORESET 0 occupies 2 symbols $(\# 0,1)$ if $\mathrm{LTE} C \mathrm{CFI}=1$, symbol 2 if $\mathrm{LTE}$ CFI $=2$, and symbol 5 if LTE CFI $=2$. In this paper, NR CORESET 0 is set to occupy symbol 2 and LTE CFI is set to be 2 in this subframe accordingly. Meanwhile, the eNB does not allocate the resource occupied by CORESET 0 to LTE PDSCH.

\subsection{Interference Avoidance Principle for LTE PBCH/SSS/PSS.} LTE PBCH, SSS, and PSS are transmitted on fixed position in both the frequency domain and time domain.

$\mathrm{PBCH}$ occupies the center 72 subcarriers in the frequency domain and the first symbols $(\# 0,1,2,3)$ of slot 2 . Its periodicity is $10 \mathrm{~ms}$.

PSS and SSS occupy the center 62 subcarriers in the frequency domain. PSS is transmitted on the last symbol of slot 0 and slot 10 in a LTE subframe while PSS is transmitted on the next to last symbol of slot 0 and slot 10 in a LTE subframe.

Based on Table 1, NR SSB, RMSI, and TRS collide with LTE PBCH, SSS, and PSS. 


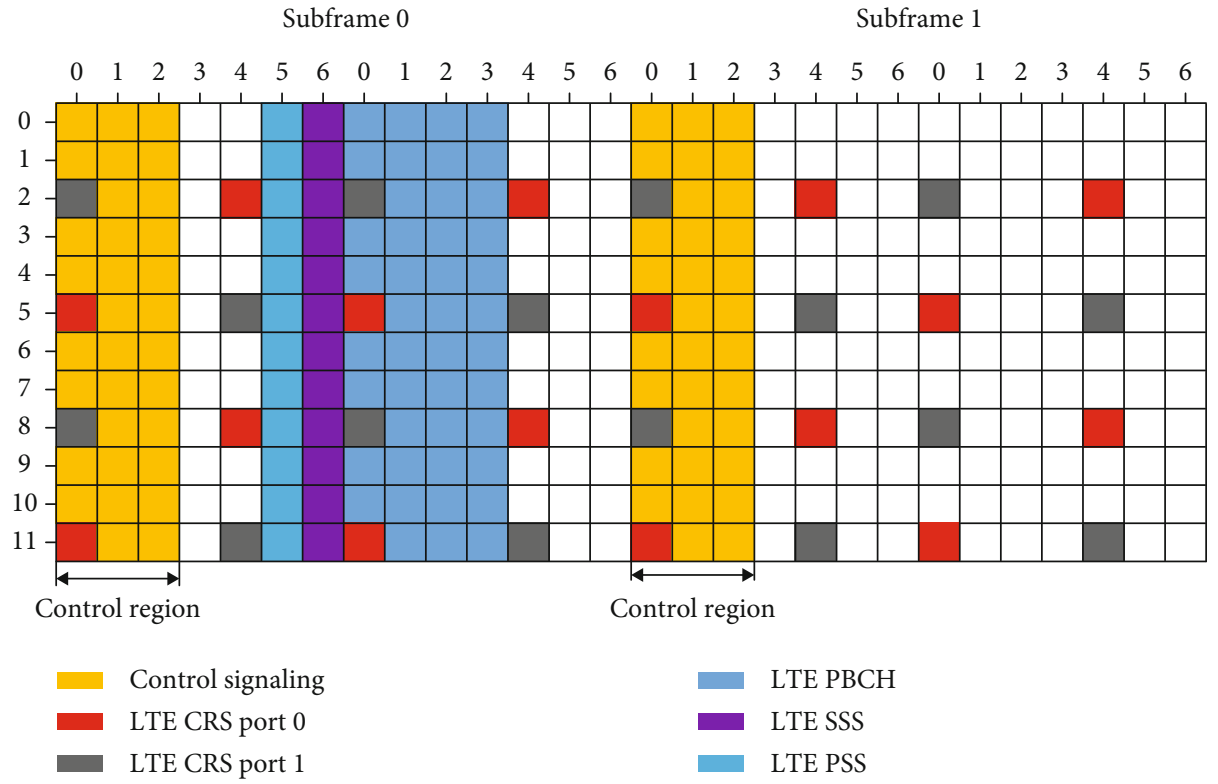

Figure 1: Symbol index of LTE control region.

NR SSB stands for Synchronization and Signal Block and consists of PBCH, PSS, and SSS. SSB occupies 4 symbols in the time domain and 240 subcarriers in the frequency domain. Its periodicity varies from $5 \mathrm{~ms}$ to $160 \mathrm{~ms}$ due to different SCSs. In this paper, low bands with bandwidth of $10 \mathrm{MHz}, 15 \mathrm{MHz}$, and $20 \mathrm{MHz}$ are focused and are below $3 \mathrm{GHz}$. The frequency position of SSB follows the rule of Case A; correspondingly, it occupies symbol 2, 3, 4, 5 or symbol $8,9,10,11$ in the time domain, and its periodicity is set to $20 \mathrm{~ms}$.

SSB fully overlaps LTE PSS if SSB is transmitted on symbol 2, 3, 4, 5 and partially overlaps LTE PBCH if it is transmitted on symbol 8, 9, 10, 11. As shown in Figure 2, the interference avoidance between NR SSB and LTE PBCH and PSS/SSS utilizes the flexible configuration of SSB in the frequency domain and the eNB also does not allocate the resource occupied by NR SSB to LTE PDSCH.

2.3. Interference Avoidance Principle for LTE CRS. As shown in Figure 2, NR SSB and RMSI collide with LTE CRS. The collision cannot be avoided in the time domain or in frequency domain. Undoubtedly, we have to choose to sacrifice LTE CRS to guarantee NR performance or sacrifice NR to guarantee LTE CRS; in other words, we only guarantee one part to transmit normally and the other part should reduce the transmission power on the collided resources. There are two options, NR puncturing for LTE CRS and LTE CRS puncturing for NR.

When NR puncturing for LTE CRS is adopted, NR reduces the transmission power on the REs colliding with LTE CRS and LTE CRS is transmitted normally.

When LTE CRS puncturing for NR is adopted, LTE reduces the transmission power on the REs of LTE CRS colliding with NR SSB/RMSI, but LTE CRS power on symbol 0 cannot be reduced with 2-port configuration and LTE CRS power on symbol $0 \& 1$ also cannot be reduced with 4-port configuration.

LTE CRS power reduction influences the reception performance of LTE UEs, DL AMC for UE should be optimized, and the eNB will reduce the MCS for UEs decoding based on LTE CRS, which power is reduced. More details can be found in Section 5 .

NR PDCCH also needs to avoid collision with LTE CRS. There are also two options, RB-level rate matching and RElevel rate matching. The performance loss of NR will be more if RB-level rate matching is adopted because LTE CRS occupies more REs and RE-level rate matching is priority to be adopted after obtaining UE capability. RE-level rate matching, LTE-CRS-ToMatchAround, is defined in "3GPP TS 38.214" [12] if the SCS of both LTE and NR is $15 \mathrm{kHz}$ and NR UE can perform RE-level rate matching on LTE CRS RE. But UE needs to report to support rateMatchingLTE-CRS; then, the procedure starts. Otherwise, RBlevel rate matching activates. If UE still does not support rateMatchingRerscSetSemiStatic, Symbol-level ZP CSI-RS is configured for UE.

2.4. Interference Avoidance Principle for PDSCH. Tracking reference signal is essential for NR, and it is used to estimate large-scale parameters, such as frequency offset, time offset, and time-frequency extension $[9,12]$. For FR1, TRS has the following characteristics:

Each CSI-RS resource only needs 1 port, and density is 3 .

The bandwidth is the minimum value of 52 and $N_{\mathrm{RB}}^{\mathrm{BWP}, i}$ or equals $N_{\mathrm{RB}}^{\mathrm{BWP}, i}$.

The frequencyDomianAllocation is fixed in row 1 . Three REs of $1 \mathrm{RB}$ on row 1 transmit CSI-IS, and 4 bits are for 4 values of $k_{0}$ in table and mapped to $4 \mathrm{RE}$ samples.

The periodicity of TRS is $80 \mathrm{~ms}$, and it occupies 4 symbols in 2 slots in case that it is configured with 52 RBs. 


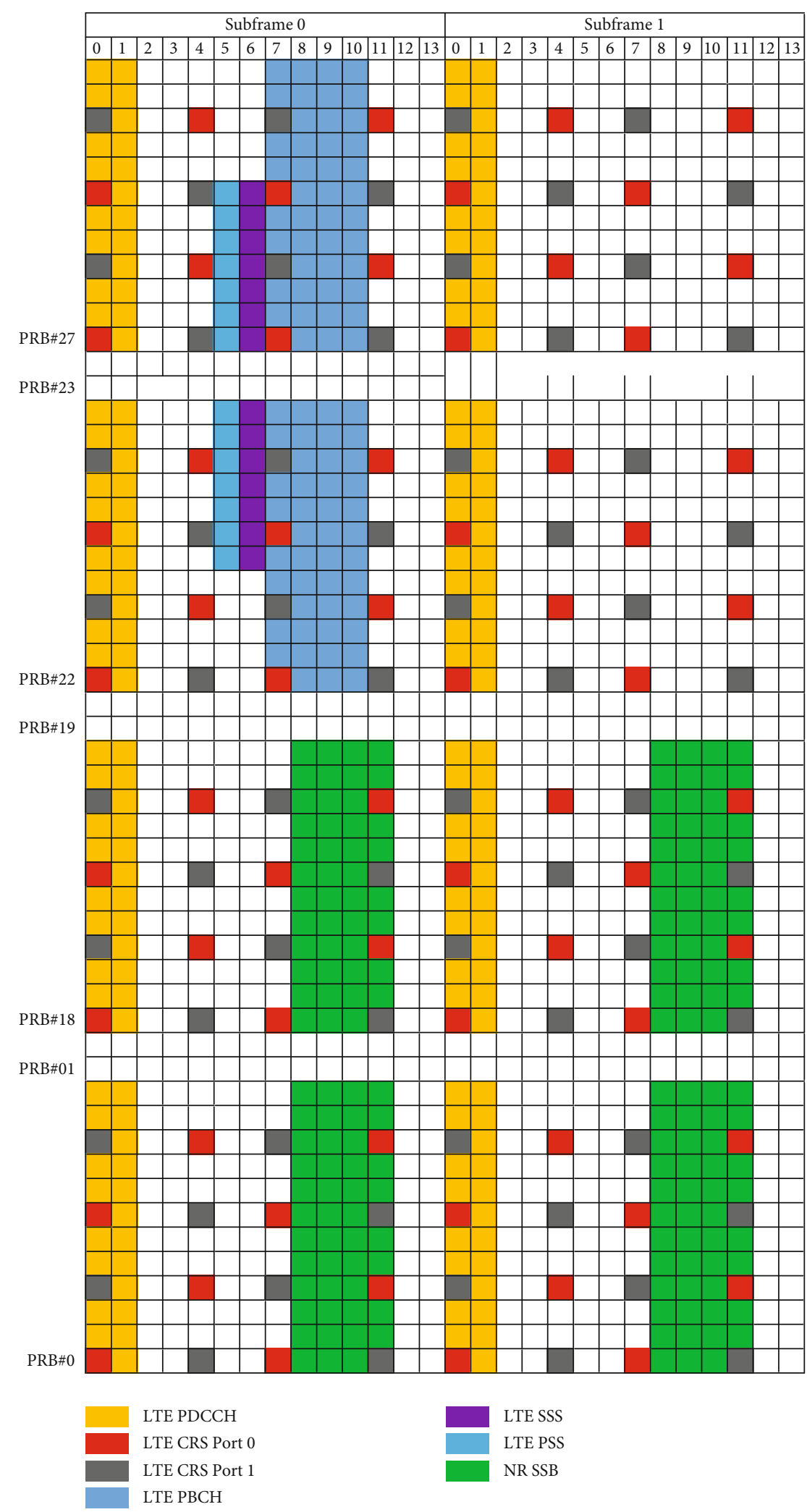

FIGURE 2: DSS symbol index for LTE and NR with SSB SCS $15 \mathrm{kHz}$ Case A. 


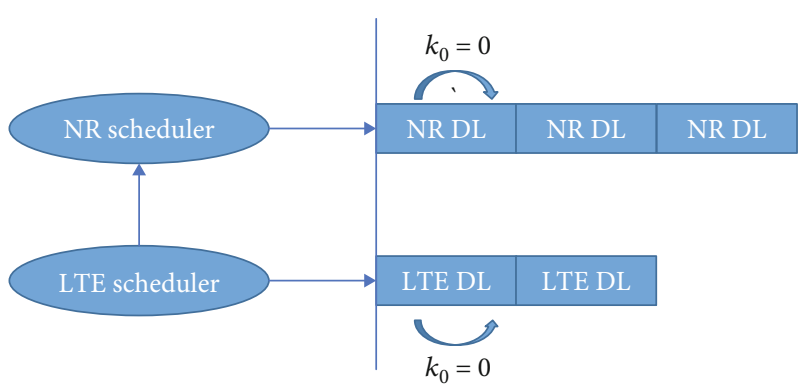

FIgURE 3: Dynamic schedule for LTE PDSCH and NR PDSCH.

The way of LTE avoiding conflict with TRS is that LTE performs conservative scheduling on TRS slot and punches on TRS REs. Meanwhile, TRS also needs to avoid conflicts with NR subframes of SSB, RMSI, and NR CSI-RS and LTE subframes of PBCH, PSS, SSS, and CRS symbols

DSS requires close cooperation between LTE and NR schedulers. LTE scheduler synchronizes LTE scheduling results to NR scheduler every TTI. NR occupies LTE remaining resources and finally merges IQ data of LTE and NR in the same air interface to realize LTE and NR sharing.

For the PDSCH of NR and LTE, the dynamic frequency resource sharing strategy is adopted. LTE transmits the scheduling results to the NR scheduler. The interaction between the downlink scheduler is shown in Figure 3. PDSCH of LTE and PDSCH of NR are shared dynamically.

\section{Uplink Interference Avoidance Principle}

The uplink interference of DSS is shown in Table 2. LTE physical channels and signals interfered by NR are PUSCH, PUCCH, SRS, and PRACH [9]. NR physical channels and signals interfered by LTE are the same as those by LTE [10]

3.1. Interference Avoidance Principle for PUCCH. LTE PUCCH occupies all 14 symbols. Although NR PUCCH adopts long or short formats and occupies less symbols, NR PUCCH also is recommended to occupy all 14 symbols to ensure its performance. Therefore, PUCCH resources cannot be reused and need to reserve resources for PUCCH.

The available RBs of $20 \mathrm{MHz}$ NR can reach $106 \mathrm{RBs}$, and 6 extra RBs can be configured as NR PUCCH. If it is still enough, NR PUCCH can be configured on RBs from both sides to the middle in turn. LTE PUCCH can be configured from the end position of NR PUCCH. The resources preempted by NR are configured by LTE PUCCH blanking.

3.2. Interference Avoidance Principle for SRS. NR SRS occupies 1,2, or 4 consecutive symbols of the last 6 symbols on slot in the time frequency, and it is limited to the last symbols for DSS. The mechanism is as follows:

\section{(1) LTE SRS is not essential}

In LTE side, cell-level SRS is configured, but UE-level SRS is not configured; LTE PUSCH avoids the symbol of cell-level SRS. In NR side, NR UE-level SRS is assigned on
TABLE 2: Uplink interference conflict between FDD LTE and NR.

\begin{tabular}{lccccc}
\hline NR/LTE & PUSCH & PUCCH & DMRS & SRS & PRACH \\
\hline PUSCH & $\sqrt{ }$ & $\sqrt{ }$ & & $\sqrt{ }$ & $\sqrt{ }$ \\
PUCCH & $\sqrt{ }$ & $\sqrt{ }$ & & $\sqrt{ }$ & $\sqrt{ }$ \\
DMRS & & & & & \\
SRS & $\sqrt{ }$ & $\sqrt{ }$ & & $\sqrt{ }$ & $\sqrt{ }$ \\
PRACH & $\sqrt{ }$ & $\sqrt{ }$ & & $\sqrt{ }$ & $\sqrt{ }$ \\
\hline
\end{tabular}

the resource of LTE cell-level SRS. NR PUSCH avoids the symbol of cell-level SRS.

\section{(2) LTE SRS is essential}

In LTE side, cell-level SRS is still configured. The resource for LTE cell-level is assigned on the basis of SRS allocation ration of LTE and NR. LTE UE-level SRS is allocated on the resource of LTE cell-level SRS. LTE also informs NR with LTE SRS resource and NR cell-level SRS resource. LTE PUSCH avoids the symbol of cell-level SRS. In NR side, NR UE-level SRS is allocated on the assigned resource of NR cell-level SRS. NR PUSCH avoids the symbol of cell-level SRS.

3.3. Interference Avoidance Principle for PRACH. For PRACH, both NR and LTE occupy partial RBs periodically and the initial position of the occupied RB can be configured. Therefore, the PRACH conflict can be avoided in the frequency domain. If both PRACH subframes do not conflict and are transmitted in different times, LTE and NR PRACH can be overlapped in the frequency domain.

3.4. Interference Avoidance Principle for PUSCH. Dynamic Resource Sharing are adopted for PUSCH and LTE scheduler informs NR scheduler of scheduled results. Figure 4 shows the interaction between NR and LTE.

$k_{2}$ stands for the subframe spacing from uplink DCI and PUSCH. The value of $k_{2}$ is 2 in NR, and the default value of $k_{2}$ is 4 in LTE.

\section{Performance Analysis on NR Puncturing for LTE CRS}

When SSB and RMSI are transmitted, UE still does not connect to gNB or eNB and obtain the rate-matching capability of LTE CRS. NR can adopt puncturing on the position of LTE CRS to protect NR SS/PBCH and RMSI from the interference from LTE CRS. But NR UE does not know NR puncturing to avoid LTE CRS; it may affect SS/PBCH and RMSI.

Figure 5 shows the position of SSB. SSB occupies 20 RBs in the frequency domain and symbol 2 to 5 or symbol 8 to 11. SSB occupies symbol 8 to 11 in DSS.

4.1. Interference Performance Simulation on SSB. There are two channel models for NR in 3GPP protocols: Tapped Delay Line (TDL) and Clustered Delay Line (CDL) [13]. CDL is mostly used for millimeter wave and Massive MIMO and TDL suitable for our simulations. TDL has 5 channel models, TDL-A, TDL-B, TDL-C, TDL-D, and TDL-E. 


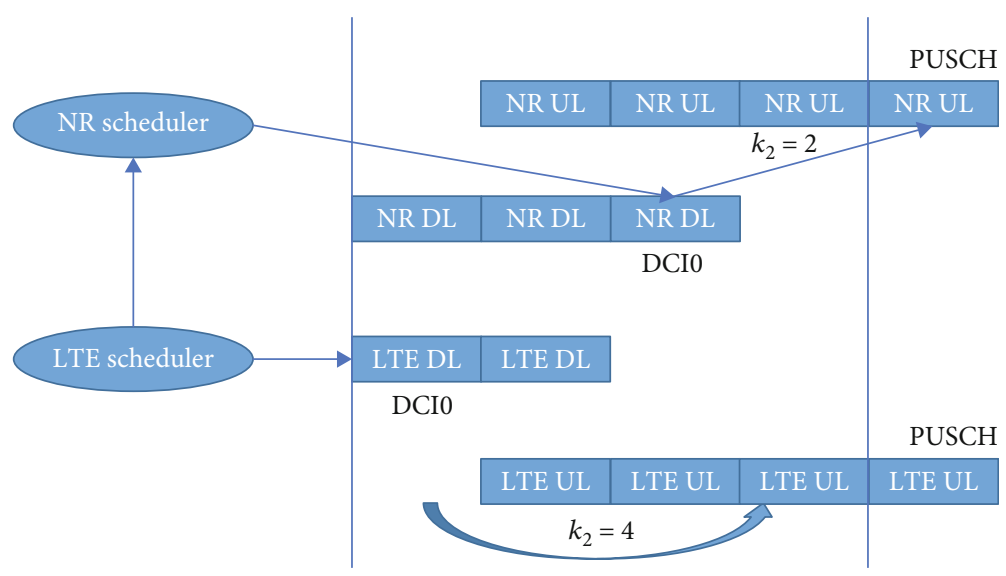

Figure 4: Dynamic schedule for LTE PUSCH and NR PUSCH.

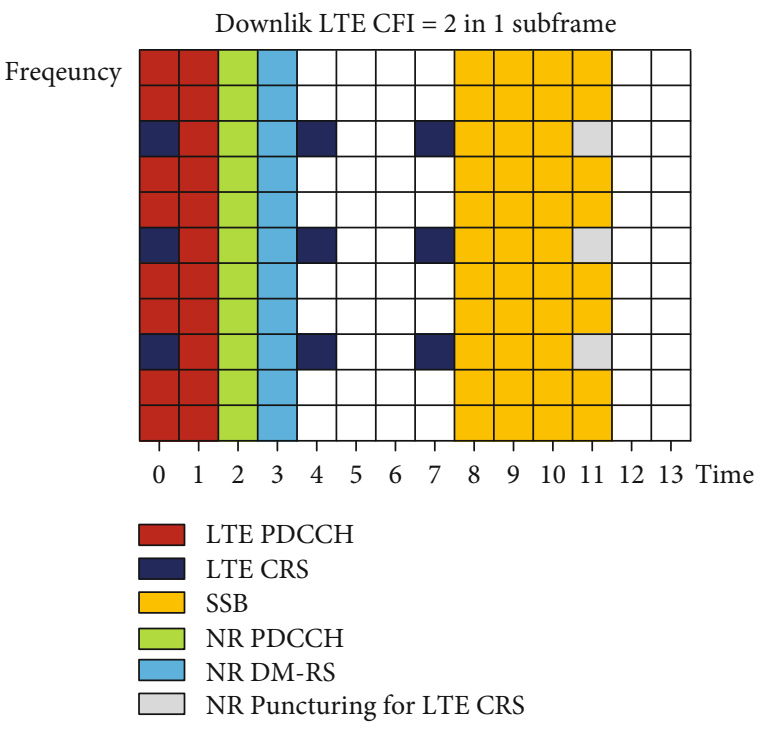

FIGURE 5: SSB allocation on FDD LTE frame.

TDL-A, TDL-B, and TDL-C belong to Non Line of Sight (NLOS), and TDL-C and TDL belong to Line of Sight (LOS). TDL-A is used for the simulation in this paper and detailed parameters can be founded in 3GPP protocol. Meanwhile, the channel model of Additive White Gaussian Noise (AWGN) is also used for our analysis.

Four scenarios are to be considered, and the basic configuration can be found in Table 3:

(1) No punch: there is no puncturing on LTE CRS.

(2) Punch type1: there is no interference on puncturing position for LTE CRS with 2 ports.

(3) Punch type2: there is no interference on puncturing position for LTE CRS with 4 ports.

(4) Interfer type1: there is load power on puncturing position for LTE CRS on with 2 ports.

(5) Interfer type2: there is load power on puncturing position for LTE CRS with 4 ports.
Based on Figure 6, the following conclusions are compared to no puncturing and puncturing with interference on LTE CRS:

(1) In AWGN simulation results, NR puncturing needs to improve by about $1.4 \mathrm{~dB}$ to obtain the same BLER. In other words, the performance loss of demodulation is about $1.4 \mathrm{~dB}$

(2) In TDL-A simulation results, NR puncturing needs to improve by less than $1 \mathrm{~dB}$ to obtain the same BLER. In other words, the performance loss of demodulation is less than $1.4 \mathrm{~dB}$

(3) In 2-port scenario, LTE CRS does not interfere PSS and SSS

(4) In 4-port scenario, LTE CRS interferes PSS and does not inter SSS

4.2. Interference Performance Simulation on RMSI. The performance estimation of the LTE CRS's impact on RMSI is to compare the demodulation difference of different Modulation and Coding Scheme (MCS) with specific BLER. Here, TDL-A and AWGN are still used as channel models and scenarios are still the same as performance analysis on SSB as shown in Table 3. The RB number of RMSI is also considered, $24 \mathrm{RBs}$ and $48 \mathrm{RBs}$, and the value of BLER is set as $10 \%$.

Based on Tables 4-7, the maximum MCS available for NR RSMI is set to 6 or 7 with 2 ports and the existing interference from LTE CRS and the maximum MCS available for NR RSMI is set below 5 with 4 ports and the existing interference from LTE CRS.

\section{Performance Analysis on LTE CRS Puncturing for NR}

LTE CRS puncturing for NR is used to protect NR SSB and RMSI from LTE CRS's interference. But LTE UE does not know LTE CRS puncturing, and it may influence RSRP measurement, demodulation, and the measurement 
TABLE 3: Basic configurations.

\begin{tabular}{lcccc}
\hline & Value & NR & RB no. & Periodicity (ms) \\
\hline Frequency & $2.1 \mathrm{GHz}$ & SSB & 20 & 20 \\
Bandwidth & $20 \mathrm{MHz}$ & RMSI & 24 & 40 \\
Subcarrier & $15 \mathrm{kHz}$ & NR SI-1\&SI-2 & 24 & 640 \\
Symbol no. & 14 & PDCCH\&PDSCH for MSGE2/4 & 24 & 320 \\
Modulation & QPSK & Paging & & \\
Channel model & AWGN TDL-A (100 ns) & & \\
UE speed & $3 \mathrm{~km} / \mathrm{h}$ & &
\end{tabular}
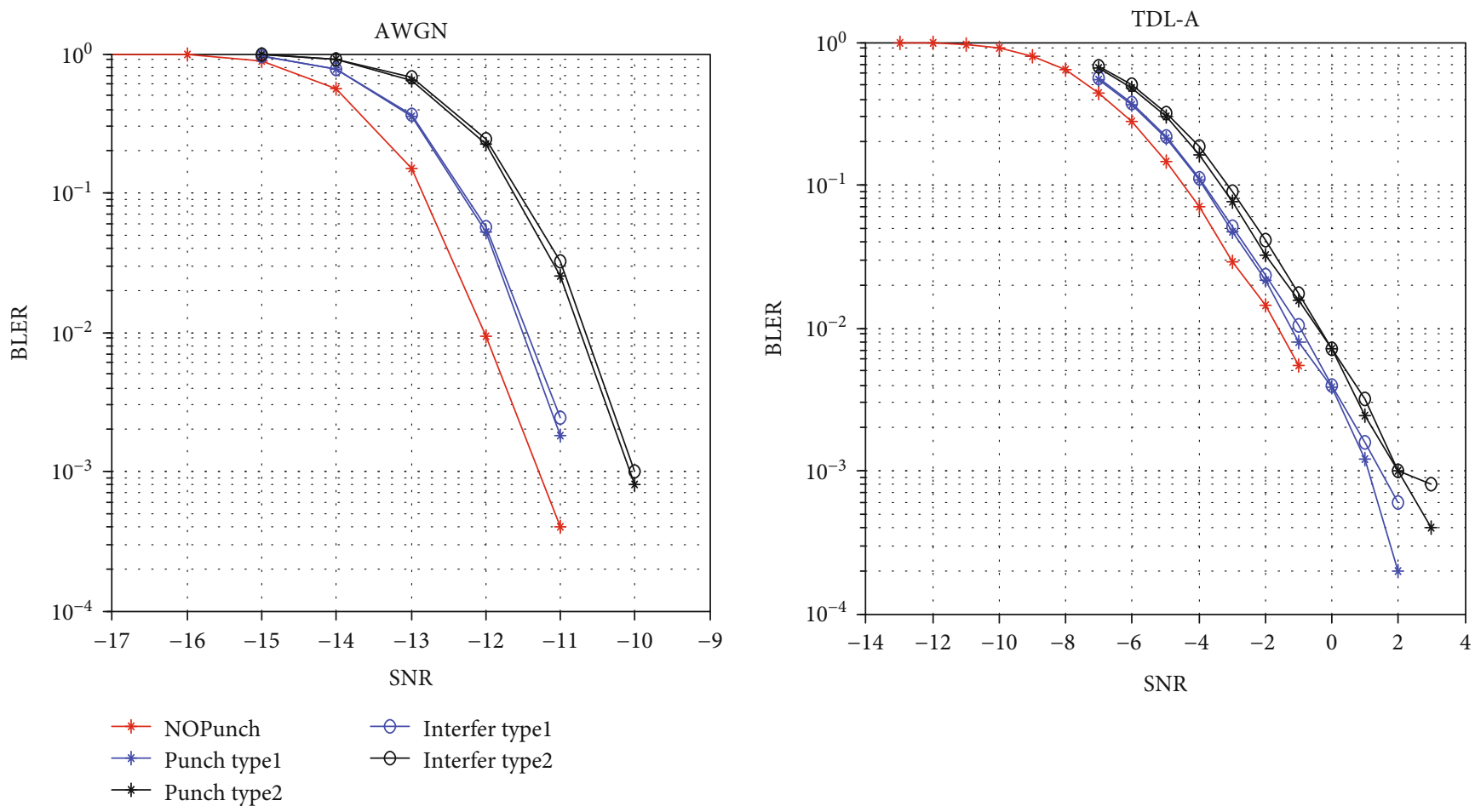

Figure 6: Simulation results based on AWGN and TDL-A.

of frequency/time offset. The above influence mentioned is presented in this part.

5.1. Interference Performance on RSRP Measurement. For RSRP determination, LTE UE may measure the cellspecific signal only from port 0 or both port 0 and port 1 . In this paper, we assume UE measures the cell-specific signal only from port 0 [14]. In addition, UE launches the filter procedure for RSRP as shown in Figure 7 [15], Layer 1 filtering is implemented by UE, and the filter coefficients of Layer 3 filtering are set by eNB. The default value is 0.5 .

Another issue is the measurement bandwidth of RSRP. The measured bandwidth of allowedMeasBandwidth is defined in SIB3 and SIB5, which is configured for neighboring cell. As with the RSRP measurement bandwidth of own cell, the configuration of allowedMeasBandwidth is not limited by the protocol and the measurement bandwidth of RSRP in SIB3 and SIB5 is modified to 6 RBs while LTE CRS puncturing is enabled.
TABLE 4: Simulation results under AWGN with 24 RB RMSI.

\begin{tabular}{lcccccc}
\hline SNR & 0 & 5 & 6 & 7 & 8 & 9 \\
\hline $\begin{array}{l}\text { No punch } \\
\text { Punch type1 }\end{array}$ & -12.37 & -8.13 & -7.23 & -6.42 & -5.69 & -4.82 \\
$\begin{array}{l}\text { Difference value for } \\
\text { type1 }\end{array}$ & 1.30 & 1.54 & 1.69 & 2.14 & 2.66 & 4.46 \\
$\begin{array}{l}\text { Punch type2 } \\
\begin{array}{l}\text { Difference value for } \\
\text { type2 }\end{array}\end{array}$ & -10.38 & -5.56 & -3.97 & -1.90 & 4.99 & $\infty$ \\
\hline
\end{tabular}

For UE, the simulation follows the above assumption. As the systematic configuration of LTE and NR, it still follows table and table.

The principles of LTE CRS puncturing are as follows:

(1) In 2-port scenario, symbol 0 is reserved for LTE PDCCH and eNB only punches on symbol 4, 7 , and 11 in partial frequency 
TABLE 5: Simulation results under AWGN with 48 RB RMSI.

\begin{tabular}{lcccccc}
\hline SNR & 0 & 5 & 6 & 7 & 8 & 9 \\
\hline $\begin{array}{l}\text { No punch } \\
\text { Punch type1 }\end{array}$ & -12.43 & -8.14 & -7.40 & -6.61 & -5.71 & -4.76 \\
$\begin{array}{l}\text { Difference value for } \\
\text { type1 }\end{array}$ & 1.24 & 1.44 & 1.67 & 1.88 & 2.51 & $\infty$ \\
$\begin{array}{l}\text { Punch type2 } \\
\begin{array}{l}\text { Difference value for } \\
\text { type2 }\end{array}\end{array}$ & -10.61 & -5.50 & -4.15 & -2.22 & 3.97 & $\infty$ \\
\hline
\end{tabular}

TABLE 6: Simulation results under TDL-A with 24 RB RMSI.

\begin{tabular}{lcccccc}
\hline SNR & 0 & 5 & 6 & 7 & 8 & 9 \\
\hline No punch & -3.66 & 0.83 & 1.70 & 2.55 & 3.35 & 4.39 \\
Punch type1 & -2.28 & 2.60 & 3.62 & 5.61 & 6.92 & $\infty$ \\
Difference value for type1 & 1.38 & 1.77 & 1.93 & 3.06 & 3.57 & $\infty$ \\
Punch type2 & -1.73 & 3.97 & 6.23 & 10.73 & $\infty$ & $\infty$ \\
Difference value for type2 & 1.93 & 3.14 & 4.57 & 8.18 & $\infty$ & $\infty$ \\
\hline
\end{tabular}

(2) In 4-port scenario, symbol 0 and 1 is reserved for LTE PDCCH and eNB only punches on symbol 4, 7,8 , and 11 in partial frequency

The simulation conditions are as follows:

(1) $\mathrm{SNR}=[3.5,0,-3,-6.5,-9.5]$ and time period $=500$ TTIs; output the instantaneous value of CRS receiving power for each TTI

(2) Punch on the position of LTE CRS; no data is transmitted. Time period $=500$ TTIs. Output the instantaneous value of CRS receiving power for each TTI.

The principles of RSRP measurement are as follows:

After linear averaging of RSRP on symbol 0, 4, 7, and 11, obtain the maximum value of these averages and it is the value of RSRP of the RB. Then, we carry on another linear averaging values of $100 \mathrm{RBs}$ of each TTI, and this value is the RSRP for current TTI. After the filter procedure with 0.5 filter coefficient, we obtain the report value of RSRP for each TTI.

As shown in Figure 8, RSPR values are presented in different path losses. "RSRP no Punch" means that LTE CRS transmits normally and does not reduce power with 2 ports. "RSRP 1+1 Punch" means that LTE CRS puncturing activates with 2 ports. "RSRP $1+1$ Punch" means that LTE CRS puncturing activates with 4 ports.

Based on the results, the delta between "RSRP no Punch" and LTE CRS puncturing does not exceed $1 \mathrm{~dB}$. If layer $1 \mathrm{fil}-$ ter also works, the delta will be further narrowed.

5.2. Performance Analysis on Demodulation. LTE CRS puncturing causes downlink capacity loss, and AMC optimization on puncturing REs is performed to reduce the loss. The systematic configuration of LTE and NR still follow the above, but RMSI configuration adopts 2 configuration's, $24 \mathrm{RBs}$ and $48 \mathrm{RBs}$. LTE transmission is TM with $\mathrm{CFI}=1$.
TABLE 7: Simulation results under TDL-A with 48 RB RMSI.

\begin{tabular}{lcccccc}
\hline SNR & 0 & 5 & 6 & 7 & 8 & 9 \\
\hline No punch & -3.92 & 0.70 & 1.45 & 2.19 & 3.22 & 4.56 \\
Punch type1 & -2.53 & 2.48 & 3.28 & 4.65 & 7.11 & $\infty$ \\
Difference value for type1 & 1.40 & 1.81 & 1.83 & 2.46 & 3.89 & $\infty$ \\
Punch type2 & -1.92 & 3.79 & 5.47 & 8.90 & $\infty$ & $\infty$ \\
Difference value for type2 & 2.00 & 3.12 & 4.02 & 6.70 & $\infty$ & $\infty$ \\
\hline
\end{tabular}

The simulation results in Table 8 show that the closer the eNB, the greater the loss caused by LTE CRS puncturing and the more the RB occupies, the greater the loss caused by LTE CRS puncturing. The capacity loss caused by LTE CRS puncturing is within $3 \%$ to $4 \%$.

5.3. Performance Analysis on Time/Frequency Offset Measurement. The simulation of frequency offset for LTE puncturing is as follows:

(1) The original frequency offset is set to $100 \mathrm{~Hz}$, and the frequency offset is estimated based on CRS then compares the difference between the estimated value and the real frequency offset

(2) LTE CRS has 200 points on symbol 0, 4, 7, and 11 of each subframe with $20 \mathrm{MHz}$ bandwidth. The frequency offset is estimated according to two pairs of symbol $0 \& 7$ and symbol $4 \& 11$ with a total of 400 points

(3) NR RMSI occupies 48 RBs, and LTE CRS is interfered on these $48 \mathrm{RBs}$ for RMSI. The interference of $-3 \mathrm{~dB}, 0 \mathrm{~dB}$, and $3 \mathrm{~dB}$ is, respectively, loaded LTE CRS on these $48 \mathrm{RBs}$

Figure 9 shows the CDF curve of the frequency offset with different interference loadings.

The estimated value of frequency offset is converged to $100 \mathrm{~Hz}$, and the probability of estimated value is less than $50 \mathrm{~Hz}$, and more than $150 \mathrm{~Hz}$ is almost 0 . Therefore, the residual frequency offset is less than $50 \mathrm{~Hz}$.

The interferences on the points of $48 \mathrm{RBs}$ are random. The theoretical value is converged to 0 after conjugate averaging. The real effective estimation of frequency offset on the other 52 RBs still dominates.

This kind of frequency offset measurement error caused by interference is occasionally seen within $20 \mathrm{~ms}$ and can be smoothly converged through UE. LTE downlink is fixed with CRS of 4 symbols, and the channel estimation of UE is interpolated in the time domain, so it is considered that it can at least resist the frequency offset within $50 \mathrm{~Hz}$. Because LTE puncturing and NR data filling lead that the estimation error is too large occasionally. After the smoothing mechanism of UE frequency offset, at least the variance will be reduced, and then the time domain interpolation of channel estimation is carried out. Therefore, the demodulation effect of frequency offset estimation caused by interference is limited. 


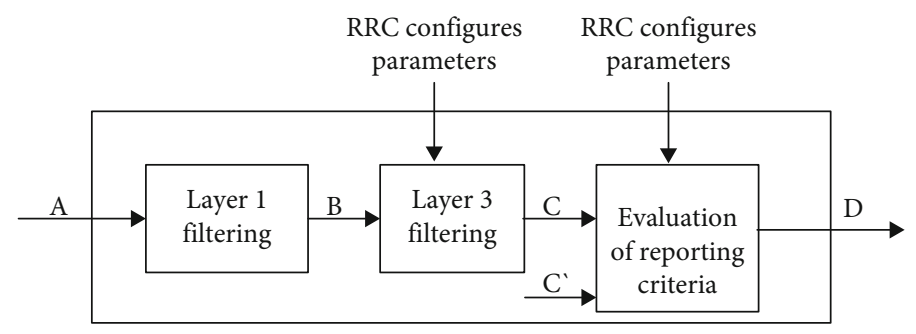

FIgURE 7: Filter procedure.

DSS, LTE, PL $=128 \mathrm{DB}$

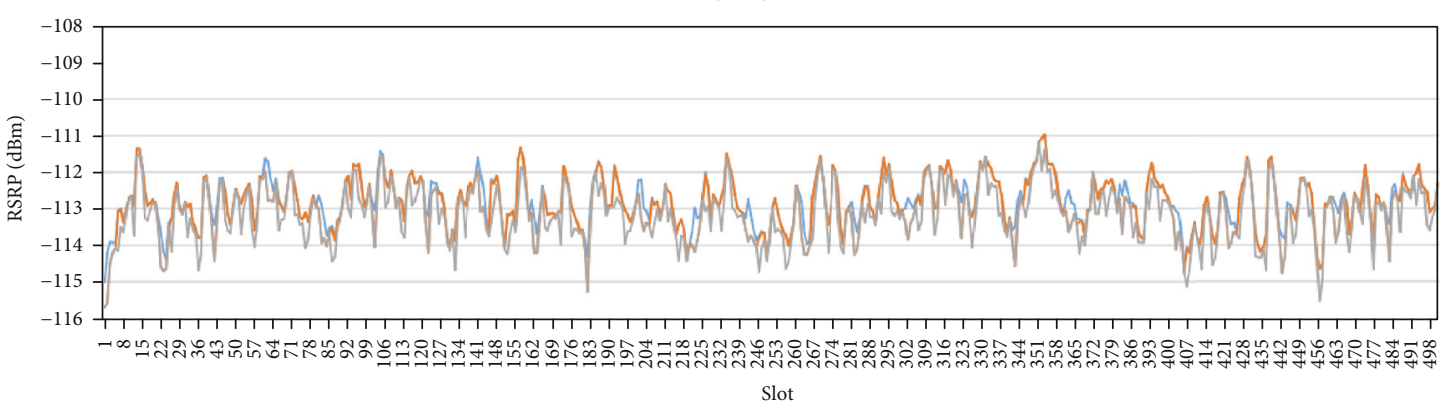

DSS, LTE, PL $=136 \mathrm{~dB}$

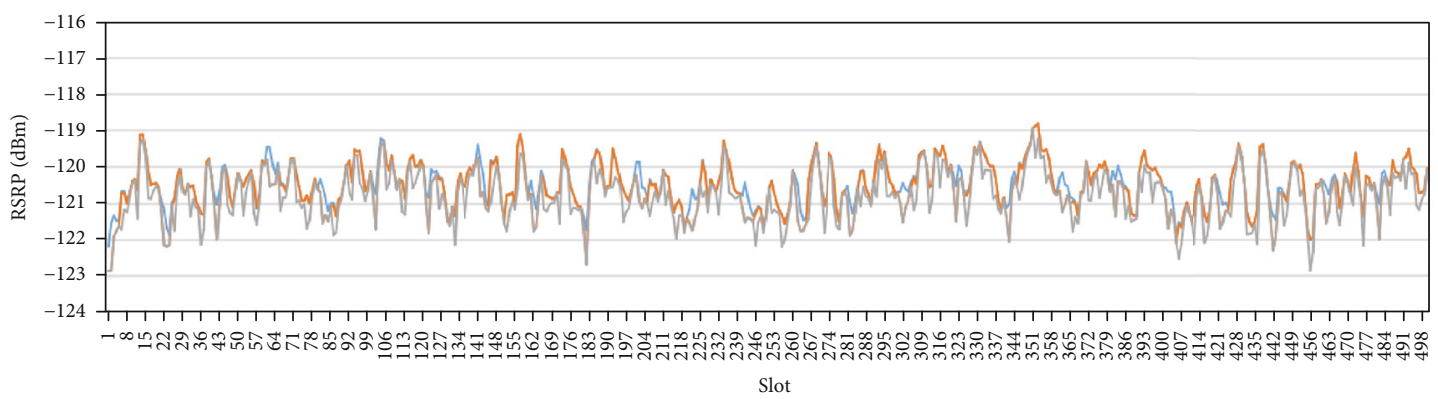

DSS, LTE, PL $=144.5 \mathrm{~dB}$

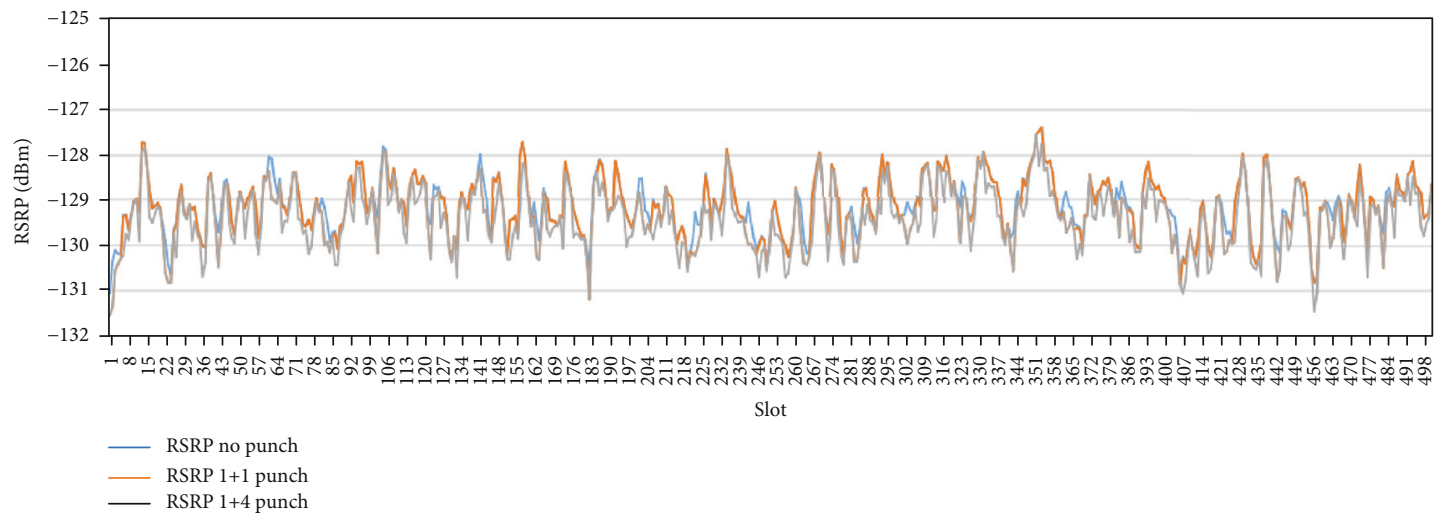

FIgURE 8: RSRP simulation with different path losses.

As with time offset, there is no impact because there is no LTE puncturing on symbol 0 and 1 .

\section{Capacity Loss Analysis Based on Systematic Overhead}

LTE CRS puncturing for NR is used to protect NR SSB and RMSI from LTE CRS's interference. But LTE UE does not
TABLE 8: Performance loss (\%) results of LTE CRS puncturing for RMSI.

\begin{tabular}{lcccccccc}
\hline SNR & -10 & -5 & 0 & 5 & 10 & 15 & 20 & 25 \\
\hline 24 RBs RSMI & 0.0 & 0.1 & 0.39 & 0.65 & 0.71 & 1.02 & 1.33 & 1.51 \\
48 RBs RMSI & 0.00 & 0.40 & 0.53 & 1.6 & 1.97 & 2.01 & 2.08 & 2.10 \\
\hline
\end{tabular}




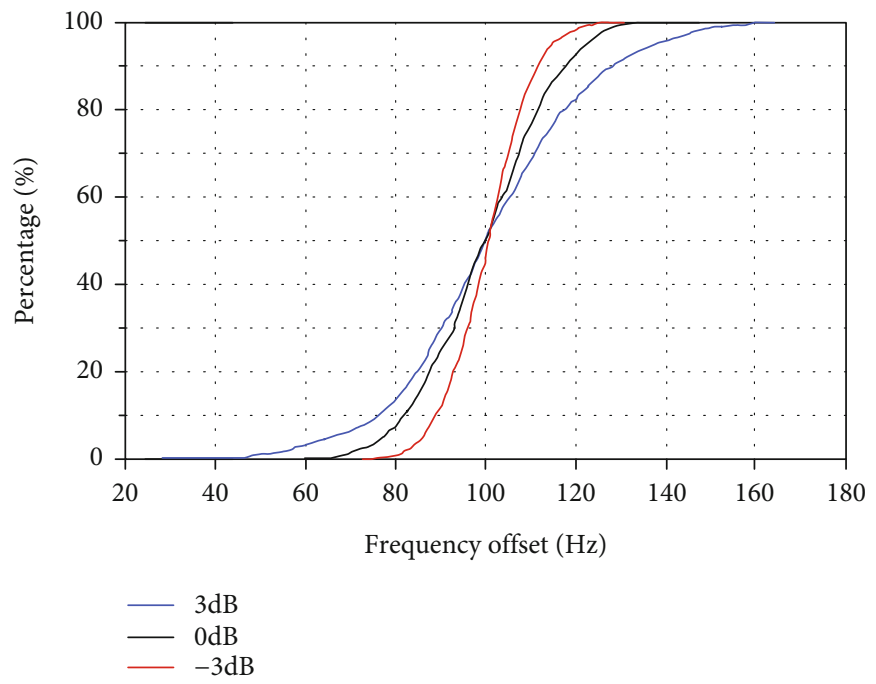

FIGURE 9: RSRP CDF curve.

TABLE 9: NR physical channel configuration for $20 \mathrm{MHz}$ bandwidth.

\begin{tabular}{|c|c|c|c|c|}
\hline \multicolumn{2}{|c|}{ NR Physical Channel } & \multirow{2}{*}{$\begin{array}{c}\text { RB number } \\
20\end{array}$} & \multirow{2}{*}{$\frac{\text { Periodicity }(\mathrm{ms})}{20}$} & \multirow{2}{*}{$\begin{array}{c}\text { Capacity loss ratio } \\
1.00 \%\end{array}$} \\
\hline \multirow{5}{*}{ Downlink } & SSB & & & \\
\hline & RMSI & 24 & 40 & $1.20 \%$ \\
\hline & NR SI-1\&SI-2 & 48 & 640 & $0.15 \%$ \\
\hline & PDCCH\&PDSCH for MSGE2/4 & 24 & 10 & $4.8 \%$ \\
\hline & Paging & 24 & 320 & $0.15 \%$ \\
\hline \multirow{2}{*}{ Uplink } & PUCCH & 1 & 1 & $1.14 \%$ \\
\hline & PRACH & 6 & 10 & $0.68 \%$ \\
\hline
\end{tabular}

know LTE CRS puncturing, and it may influence RSRP measurement, demodulation, and the measurement of frequency/time offset. The above influences mentioned are presented in this part.

Undoubtedly, the LTE capacity will be reduced when DSS activates due to the introduction of NR physical channels and signals. The percentage of NR physical channels and signals can be calculated as follows:

CapacityLossRatio $=\frac{\text { ChannelRBNum } *(\text { TransmitTime } / \text { ChannelPeriodicity })}{\text { SystemRBNum } * \text { TransmitTime }}$,

where SystemRBNum is the whole RB number of LTE carrier, ChannelRBNum is the RB number occupying the corresponding physical channels, ChannelPeriodicity is the channel's periodicity, and TransmitTime is the period of data.

Table 9 shows the capacity loss of $20 \mathrm{MHz}$ carrier with DSS. The downlink capacity loss reaches $7.3 \%$, and the downlink capacity loss reaches $1.82 \%$.

\section{Conclusion}

However, the flexible configuration of NR physical layer gives the opportunity to make FDD LTE and NR coexist in the dedicated carriers, but the collisions occurring in DSS cannot be avoided without performance loss and the performance loss caused by collisions with LTE CRS; the performances are acceptable. On the other hand, the essential overhead for DSS also brings the capacity loss and reduces the essential overhead based on the flexible design on NR physical layer as soon as possible.

Anyway, this paper gives a comprehensive principle on dynamic spectrum sharing between LTE and NR and detailed explanations on the implementation of physical layer for DSS. The corresponding simulations undoubtedly have a significance on the research and commercial deployment of DSS in the existing network.

In the meanwhile, there are still some limitations and challenges. Our simulations just focus on a single cell. In the real network, the density of cells is very close and the wireless environment is more complicated. The intercell interference will be the biggest challenge for the commercial deployment. The intercell interference avoidance will be our next target.

\section{Data Availability}

The datasets used and/or analyzed during the current study are available from the corresponding author on reasonable request. 


\section{Conflicts of Interest}

The authors declared no potential conflicts of interest with respect to the research, authorship, and/or publication of this article.

\section{Acknowledgments}

The authors are supported both by Shenzhen Fundamental Research Program under grant number 20210317191843003 and Shanxi Provincial Key R\&D under grant number 2021ZDLGY05-01.

\section{References}

[1] Huawei, "5g wireless network planning solution white paper," Available: https://www-file.huawei.com/-/media/corporate/ pdf/white\%20paper/2018.

[2] Y. Arjoune and N. Kaabouch, "A comprehensive survey on spectrum sensing in cognitive radio networks: recent advances, new challenges, and future research directions," Sensors, vol. 19, no. 1, p. 126, 2019.

[3] W. S. H. M. W. Ahmad, N. A. M. Radzi, F. S. Samidi et al., "5G technology: towards dynamic spectrum sharing using cognitive radio networks," IEEE Access, vol. 8, pp. 14460-14488, 2020.

[4] S. Maximov, Y. Chung-Cheng, and M. A. El-saidy, “5G NR and 4G LTE coexistence: a comprehensive deployment guide to dynamic spectrum sharing," MediaTek, MediaTek, 2020.

[5] G. Barb, F. Alexa, and M. Otesteanu, "Dynamic spectrum sharing for future LTE-NR networks," in Proceedings of the 2020 International Symposium on Electronics and Telecommunications (ISETC), vol. 5-6, pp. 1-4, Timisoara, Romania, 2020.

[6] N. Networks, LTE-NR DSS Phase I, Network Engineering, Espoo, Finland, 2020.

[7] ZTE and ZTE Microelectronics, Discussion on NR-LTE CoExistence, 3GPP TSG RAN WG1 Meeting\#88 R1-1701618, Athens, Greece, 2017.

[8] NR Base Station (BS), Radio Transmission and Reception, 3GPP TS 38.104 R16, 3GPP, 2020.

[9] Evolved Universal Terrestrial Radio Access (E-UTRA), Physical Channels and Modulation, 3GPP TS 36.211 R16, 3GPP, 2020.

[10] NR, Physical Channels and Modulation, 3GPP TS 38.211 R16, 3GPP, 2020.

[11] NR, Radio Resource Control (RRC), Protocol specification, 2020, 3GPP TS 38.311 R16.

[12] NR, Physical Layer Procedures for Data, 3GPP TS 38.214 R16, 3GPP, 2020.

[13] "Study on channel model for frequencies from 0.5 to $10 \mathrm{GHz}$, 3GPP TR 38.9013GPP.

[14] NR, Physical Layer Procedures for Data, 3GPP TS 38.214 R16, 3GPP, 2020.

[15] Evolved Universal Terrestrial Radio Access (E-UTRA) and Evolved Universal Terrestrial Radio Access Network (EUTRAN), Overall description; Stage 2, 3GPP TS 36.300 R16, 2020. 